Proceedings of the 2010 Winter Simulation Conference

B. Johansson, S. Jain, J. Montoya-Torres, J. Hugan, and E. Yücesan, eds.

\title{
MONTE CARLO SIMULATION-BASED SUPPLY CHAIN DISRUPTION MANAGEMENT FOR WARGAMES
}

\author{
Shilan Jin, Jun Zhuang \\ Industrial and Systems Engineering \\ SUNY at Buffalo \\ Buffalo, NY 14228, USA
}

\author{
Zigeng Liu \\ Wisconsin School of Business \\ University of Wisconsin-Madison \\ Madison, WI 53706, USA
}

\begin{abstract}
In this paper, we integrate supply chain risk management with a government-terrorist game conducted in war zones (such as Afghanistan and Iraq). The equilibrium outcomes of wargames depend on the government's resources delivered through military supply chains, which are subject to disruptions such as natural disasters and terrorism. We study the government's optimal pre-disruption preparation strategies, including inventory protection and capacity backup protection. Considering the uncertainties (e.g., the outage length of a disruption and the level of resources available to the terrorist), we conduct Monte Carlo simulation experiments to numerically investigate the benefits using our disruption preparation strategies compared with other strategies.
\end{abstract}

\section{INTRODUCTION}

Research on supply chain risk management has been popular in recent years (Juttner et al. 2003). Chopra et al. (2007) indicated the importance of separating the recurrent supply risk and disruption risk for appropriate mitigation mechanisms. Christopher and Lee (2004) argued that supply chains are affected by many factors, including the uncertain changes in business strategies, and indicated that it is easier to mitigate the supply chain risks with more information. Christopher and Peck (2004) emphasized that it is critical to understand the nature of supply chain risks while building resilient supply chain networks. Sheffi et al. (2003) studied how companies perceive, predict, and assess the risks in order to protect their supply chains accordingly.

Various risks exist in supply chain networks, including social disasters, natural disasters, and terrorism. In the mid-1990s, a severe economy recession in Honduras heavily impacted local banana industries, resulting in banana price soaring worldwidely. In late 1998, a category-5 hurricane hit the banana industry in Honduras, and more than 50\% of the banana crops were inundated by floods, which resulted in damages of \$3 billion (Encyclopedia of the Nations 2008). The 1999 Taiwan earthquake caused a significant worldwide supply shortage of Random Access Memory (RAM), and tripled unit prices, due to a six-day shutdown of RAM factories in the Taiwan area (Hopp, Liu, and Liu 2010). The 2004 Indian Ocean tsunami devastated major economy activity (primarily fisheries) in a large region of Southern Asia by destroying the commonly used fishing tackles (UK Agricultural Biodiversity Coalition 2004). During the days after 9/11/2001 terrorist attack, Ford idled several assembly lines, because its trucks were delayed at the Canadian and Mexican borders (Sheffi 2002).

Chopra and Sodhi (2004) classified supply chain risks into nine categories (disruption, delays, system risks, forecasting risks, intellectual property risks, procurement risks, receivables, inventory risks, and capacity risks) and analyzed the corresponding mitigation strategies. Hopp, Liu, and Liu (2010) built mathematical models on supply chain networks and introduced two pre-disruption protection mechanisms to mitigate supply chain risks: capacity backup (including subtree capacity protection and single-node capacity protection) and inventory protection. In this paper, we focus on the application of these two protection mechanisms against disruptions (the first category of risks classified by Chopra and Sodhi (2004) above) on the military supply chains for wargames.

Since September 11, 2001, many researchers have studied homeland security games, including wargames between attackers and defenders (Zhuang and Bier 2007). The equilibrium outcomes of wargames depend on the government's resources delivered through military supply chains to war 


\section{Jin, Liu and Zhuang}

zones, which are subject to disruptions such as natural disasters and terrorism. However, to the best of our knowledge, there are no existing studies on the integration of supply chain risk management and wargames. This paper is organized as follows: Section 2 presents two disruption preparation models (inventory protection model and capacity backup model); Section 3 presents numerical Monte Carlo simulation-based models to examine the benefits of our disruption preparation models by comparing with other strategies; and Section 4 concludes the paper and provides some future research directions.

\section{MODELING FRAMEWORK}

As suggested by Zhuang and Bier (2007), governments and terrorists jointly determine their efforts at equilibrium in wargames, and their equilibrium utilities depend on the available resources delivered to war zones, which are subject to military supply chain disruptions. In our paper, we allow the government to decide the amount of resources delivered to war zones and the investment in risk management (inventory or capacity backup) against disruptions. On the other hand, the terrorist's resource is assumed to be given exogenously, and we do not study the terrorist's risk management (partially due to their limited finance and capacities). The above assumptions are reasonable since government often has the ability to obtain needed resources (e.g., transferring funds from other government branches), while it might be difficult for many terrorist organizations to do the same.

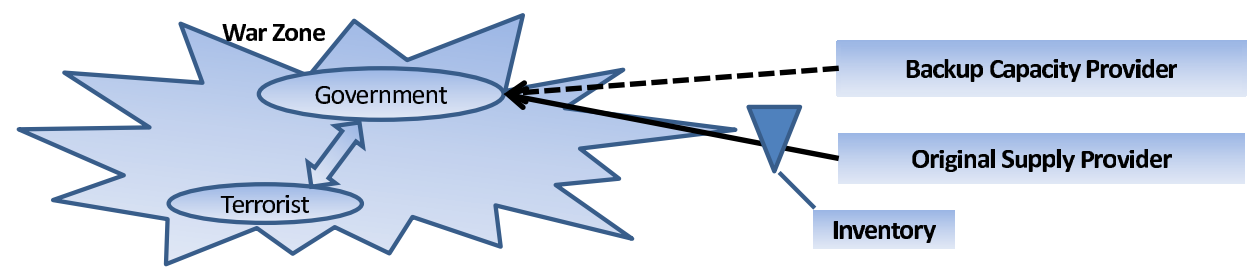

Figure 1: Integrating military supply chain risk management with wargames between a terrorist and a government

As Figure 1 shows, we consider two protection mechanisms the government may invest in: inventory protection and capacity backup protection. Both of which are interesting and realistic: after a disruption occurs, some fast, expendable resources (e.g., bullets and bombs) are difficult to supply through backup capacity providers in a timely manner, while some other resources could not be held in inventory for a long time (e.g., food and medicines). For the sake of simplicity, we consider one single generic resource and analyze these two protection mechanisms separately.

We acknowledge that, in reality, the supply chain network may be very complex (e.g., different nodes could be supported by different inventories or backup suppliers). However, in this paper, we only consider a single capacity backup provider and assume that the daily needed resource can be fully satisfied by this provider.

As Figure 2 shows, we let one period consist of peace time (which starts at the end of the last-period disruption and ends at the beginning of the current-period disruption) and failure time (which stars from the beginning of the current-period disruption and ends when the disruption influence ends). Hence, the length of each period depends on two factors: the peace time length and the failure time length.

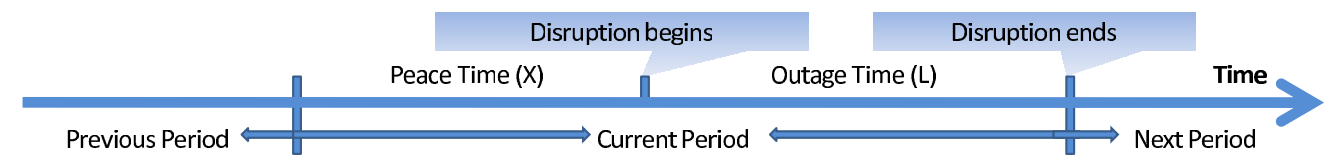

Figure 2: Periods and timing for peace, outage and disruption

Table 1 introduces the notation that is used throughout the paper. We assume that the government's optimal payoff in the war zone for each period is given by $\frac{r}{r+R} \cdot v$. This may be obtained by solving a simultaneous-more game between a government and a terrorist, whose defense and attack efforts are bounded by $r$ and $R$, respectively (Zhuang and Bier 2007). On the other hand, we consider that the government maximizes her total expected payoff in war zone, subtracting the expected cost on 
Table 1: Notation used throughout the paper.

\begin{tabular}{|c|l|r|l|}
\hline \multicolumn{2}{|l|}{ Characteristic parameters } & \multicolumn{2}{|l|}{ Characteristic parameters } \\
\hline$R$ & Resource of terrorist & $f_{D}(d)$ & Probability density function of delay time \\
$v$ & Government's asset valuation & $f_{L}(l)$ & Probability density function of failure time \\
$D$ & Time required (delay) to bring capacity backup & $f_{X}(x)$ & Probability density function of peace time \\
$L$ & Failure time in disruption & & \\
$X$ & Peace time (before disruption) & \multicolumn{2}{|c|}{ Decision Variables } \\
\hline \hline Cost parameters & $I$ & Inventory level (in time unit) \\
\hline$m$ & Daily revenue rate & $r$ & Daily resource that government uses \\
$h$ & Daily holding cost per unit inventory & & \\
$\alpha$ & Reservation cost of capacity backup & & \\
$\beta$ & Daily usage cost of capacity backup & & \\
\hline
\end{tabular}

preparation against disruptions, which is a function of the government's resource $r$ and the inventory level $I$ (for inventory protection mechanism).

\subsection{Inventory Protection Mechanism}

We first consider that the government holds a certain amount of inventory in peace time as a preparation against disruptions, and uses this inventory during the outage time (which is denoted by $L$ ). Equation (1) below shows the government's expected utility, using the inventory protection model.

During peace time, the government holds inventory $I$, costing $h \cdot I$ per day. When a disruption occurs, the inventory position decreases with a rate of $r$ and, hence, holding cost decreases with a rate of $h \cdot r$ until the inventory is empty. After the inventory is empty, we assume that the government experiences a revenue loss at a rate of $m$. Therefore, we have the total expected utility of government over one period:

$$
\begin{aligned}
z(I, r)= & \underbrace{\frac{r}{r+R} \cdot v}_{\text {payoff in war zone }}-\underbrace{h \cdot I \cdot \int_{0}^{\infty} x \cdot f_{X}(x) \cdot \mathrm{d} x}_{\text {holding cost during peace time }} \\
& -\underbrace{h \cdot \int_{0}^{\infty} \sum_{i=0}^{l-1}[I-i \cdot r]^{+} \cdot f_{L}(l) \cdot \mathrm{d} l}_{\text {holding cost during outage time }}-\underbrace{m \cdot \int_{0}^{\infty}[l \cdot r-I]^{+} \cdot f_{L}(l) \cdot \mathrm{d} l}_{\text {revenue loss during outage time }} .
\end{aligned}
$$

\subsection{Capacity Backup Mechanism}

Using the capacity backup mechanism, the government does not hold inventory but, instead, she reserves the backup supplier before disruptions and consumes the capacity backup during the outage time (after a disruption occurs). Equation (2) below provides the government's total expected utility when using capacity backup as preparation against disruptions. The government pays the reservation fee at a rate of $\alpha$ during the entire period and the usage fee at a rate of $\beta$ during the outage time. Therefore, we have the government's expected utility:

$$
\begin{aligned}
z(r)= & \underbrace{\frac{r}{r+R} \cdot v}_{\text {payoff in war zone }}-\underbrace{\alpha \cdot r \cdot\left(\int_{0}^{\infty} x \cdot f_{X}(x) \cdot \mathrm{d} x+\int_{0}^{\infty} l \cdot f_{L}(l) \cdot \mathrm{d} l\right)}_{\text {reservation cost for capacity backup }} \\
& -\underbrace{\beta \cdot r \cdot \int_{0}^{\infty} \int_{0}^{l}(l-d) \cdot f_{L}(l) \cdot f_{D}(d) \cdot \mathrm{d} d \cdot \mathrm{d} l}_{\text {usage cost for capacity backup }}-\underbrace{m \cdot r \cdot \int_{0}^{l} d \cdot f_{D}(d) \cdot \mathrm{d} d}_{\text {revenue loss during delay time }} .
\end{aligned}
$$




\section{SIMULATION ANALYSIS}

With the inventory protection / capacity backup mechanism, the government may balance the preparation cost and the failure loss in order to maximize her overall expected utility. To investigate the impact of preparation on the government's utility, we conduct a Monte Carlo simulation to examine the benefit that the government would receive when using protection strategies based on our modeling frameworks, as compared with other strategies.

\subsection{Design of Numerical Experiments}

We have designed two sets of Monte Carlo simulation experiments in order to study the inventory protection mechanism and capacity backup mechanism in Section 3.2 and 3.3, respectively. Table 2 summarizes the baseline input parameter values. In each Monte Carlo simulation experiment, we randomly generate the following four parameters: peace time $(X)$, outage length $(L)$, time required to bring capacity backup online ( $D$, for capacity backup model only), and terrorist resources $(R)$. We also assume that each of these parameters follows a triangular distribution with a lower limit (LL), a mode (MO), and a upper limit (UL) (as shown in Table 2).

Table 2: Baseline case values of inputs parameters in numerical studies.

\begin{tabular}{cc|cc|cc}
\hline Parameter & Value & Parameter & Value & Parameter & Value \\
\hline \hline$R$ & 60 & $v$ & 2700 & $m$ & 1.2 \\
$h$ & 0.006 & $\alpha$ & 0.3 & $\beta$ & 0.008 \\
$X_{L L}$ & 4 & $X_{M O}$ & 10 & $X_{U L}$ & 15 \\
$L_{L L}$ & 4 & $L_{M O}$ & 10 & $L_{U L}$ & 16 \\
$D_{L L}$ & 1 & $D_{M O}$ & 3 & $D_{U L}$ & 4 \\
$R_{L L}$ & 40 & $R_{M O}$ & 60 & $R_{U L}$ & 100 \\
\hline
\end{tabular}

\subsection{Impact of Inventory Protection Mechanism on Optimal Government's utility}

In this section, we compare the inventory protection policy based on our Inventory Protection model provided in Section 2.1 with two policies that the government may use:

- Police A: Zero inventory policy, i.e., $I=0$;

- Police B: Mean outage length inventory policy, i.e., by some means (e.g., using some historical data), the government may have an estimate of the mean outage length $\left(\mu_{\text {outage }}\right)$ and set the inventory level equal to $I=\mu_{\text {outage }} \cdot r$.

In the following, we call the above two policies Police A and Police B together as the Fixed Inventory Level Policy, and under the randomly generated scenarios of $X, L$, and $R$, we present the corresponding numerical model to calculate the government's utility $\left(z_{1}\right)$ as follows:

$$
\max _{r} z_{1}(r)=\frac{r}{r+R} \cdot v-h \cdot I \cdot X-h \cdot \sum_{i=0}^{L-1}[I-i \cdot r]^{+}-m \cdot[L \cdot r-I]^{+},
$$

where $I=0$ (zero inventory policy) or $I=\mu_{\text {outage }} \cdot r$ (mean outage length inventory policy). 


\subsubsection{Inventory Protection Numerical Model}

Based on the baseline case described in Section 3.1, Equation (1) becomes:

$$
\begin{aligned}
\max _{I, r} z_{0}(I, r)= & \frac{r}{r+R} \cdot v-h \cdot I \cdot E[X] \\
& -h \cdot\left[\sum_{l=L_{L L}}^{L_{M O}}\left(\sum_{i=0}^{l-1}[I-i \cdot r]^{+} \cdot f_{L}^{(1)}(l)\right)+\sum_{l=L_{M O}+1}^{L_{U L}}\left(\sum_{i=0}^{l-1}[I-i \cdot r]^{+} \cdot f_{L}^{(2)}(l)\right)\right] \\
& -m \cdot\left[\sum_{l=L_{L L}}^{L_{M O}}[l \cdot r-I]^{+} \cdot f_{L}^{(1)}(l)+\sum_{l=L_{M O}+1}^{L_{U L}}[l \cdot r-I]^{+} \cdot f_{L}^{(2)}(l)\right],
\end{aligned}
$$

where $E[X]=\frac{X_{L L}+X_{M O}+X_{U L}}{3}, f_{L}^{(1)}(l)=\frac{2\left(l-L_{L L}\right)}{\left(L_{U L}-L_{L L}\right)\left(L_{M O}-L_{L L}\right)}$ and $f_{L}^{(2)}(l)=\frac{2\left(L_{U L}-l\right)}{\left(L_{U L}-L_{L L}\right)\left(L_{U L}-L_{M O}\right)}$.

We denote the optimal solution pair $\left(r^{*}, I^{*}\right)$ to Equation (4) as Best Response Policy. For the baseline case (as shown in Table 2), we calculate the optimal government's policy to maximize the expected government's utility denoted as $z_{0}^{*}\left(I^{*}, r^{*}\right)$.

To examine whether the Best Response Policy performs well under randomly generated scenarios, we introduce $z_{2}^{*}$ as the government's utility with the Best Response Policy, and realized under the randomly generated scenarios of $X, L$ and $R$ :

$$
z_{2}^{*}=\frac{r^{*}}{r^{*}+R} \cdot v-h \cdot I^{*} \cdot X-h \cdot \sum_{i=0}^{L-1}\left[I^{*}-i \cdot r^{*}\right]^{+}-m \cdot\left[L \cdot r^{*}-I^{*}\right]^{+} .
$$

Under each randomly generated set of parameters $(X, L$, and $R)$, we find the optimal government's policy to maximize the government's utility $\left(z_{3}\right)$ :

$$
\max _{I, r} z_{3}(I, r)=\frac{r}{r+R} \cdot v-h \cdot I \cdot X-h \cdot \sum_{i=0}^{L-1}[I-i \cdot r]^{+}-m \cdot[L \cdot r-I]^{+} .
$$

Note that, in reality, it might be impossible for the government to know a disruption scenario $(X, L$, and $R$ ) before developing a preparation plan (the Ideal Preparation Policy), i.e., we can view $z_{3}^{*}$ as the Ideal Government's Utility.

In the following, in order to quantitatively further investigate the benefit of using the Best Response Policy we compare the Best Response Policy with the Fixed Inventory Level Policy and the Ideal Preparation Policy.

\subsubsection{Experiment Procedure for Inventory Protection Model}

The experiment is designed according to the following procedure:

Step 1: Use Equation (4) to find the government's optimal resource $r^{*}$ and the optimal inventory level $I^{*}$ for the baseline case (as shown in Table 2); record $r^{*}, I^{*}$, and the corresponding government's optimal utility $z_{0}^{*}\left(I^{*}, r^{*}\right)$.

Step 2: $\quad$ Randomly generate 100 sets of the following three parameters: $X, L$, and $R$.

Step 3: $\quad$ For each set of randomly generated parameters $(X, L$, and $R)$, calculate and record the government's utility $z_{1}^{A *} \equiv z_{1, I=0, r=\hat{r}}^{*}$, where $\hat{r}$ is the optimal solution to Equation (3) with $I=0$ obtained by using simulation optimization method as shown below:

- Sub-Step 3.1: Consider the following possible levels of $r: r^{i}=i, \forall i=0,1, \cdots, 800$.

- Sub-Step 3.2: For each level of $r, r=r^{i}, i=0,1, \cdots, 800$, under the 100 sets of three randomly generated parameters $(X, L$, and $R$ ), use Equation (3) to calculate the corresponding 100 values of $z$ , and record the average value $\bar{z}_{1, I=0, r=r^{i}}$.

- Sub-Step 3.3: Compare all $\bar{z}_{1, I=0, r=r^{i}}, i=0,1, \ldots, 800$, find $\hat{r} \equiv r^{i *}=\arg \max _{r^{i}}\left\{\bar{z}_{1, I=0, r=r^{i}}\right\}$, and record $\hat{r}$. 
Step 4: $\quad$ For each set of randomly generated parameters $(X, L$, and $R)$, calculate and record the government's utility $z_{1}^{B *} \equiv z_{1, I=\mu_{\text {outage }}^{*}, r=\tilde{r}}^{*}$, where $\tilde{r}$ is the optimal solution to Equation (3) with $I=\mu_{\text {outage }} \cdot r$ obtained by using the simulation optimization method as demonstrated in SubStep 3.1-3.3.

Step 5: $\quad$ For each set of randomly generated parameters $(X, L$, and $R)$, calculate and record the government's utility $z_{2}^{*} \equiv z_{2, I=I^{*}, r=r^{*}}$ by using Equation (5), and the government's optimal utility $z_{3}^{*}$ by using Equation (10).

Step 6: Under each set of generated parameters, calculate and record:

- The value $\omega_{A}=\left(z_{2}^{*}-z_{1}^{A *}\right) /\left|z_{1}^{A *}\right|$, and the value $\omega_{B}=\left(z_{2}^{*}-z_{1}^{B *}\right) /\left|z_{1}^{B *}\right|$, representing the percent increase of utility that the government would experience, if the government invests in inventory preparedness using the Best Response Policy, as compared to using Policy A and Policy B, which were introduced in Section 3.2.

- The value $\omega_{23}=\left(z_{2}^{*}-z_{3}^{*}\right) /\left|z_{3}^{*}\right|$, which represents the difference between the government's utility using the Best Response Policy and the Ideal Government's Utility.

Step 7: Find the sample means of $\omega_{A}, \omega_{B}$, and $\omega_{23}$, and denote them by $\bar{\omega}_{A}, \bar{\omega}_{B}$, and $\bar{\omega}_{23}$, respectively.

Step 8: $\quad$ Find the sample standard deviations of $\omega_{A}, \omega_{B}$, and $\omega_{23}$, and denote them as $s_{\omega_{A}}, s_{\omega_{B}}$, and $s_{\omega_{23}}$, respectively.

\subsubsection{Simulation Results}

The simulation results of the experiment are shown in Table 3. Figure 3 demonstrates $z_{1}^{A *}$ and $z_{1}^{B *}$ (the government's utility using the two types of Fixed Inventory Level Policy), $z_{2}^{*}$ (the government's utility using the Best Response Policy), and $z_{3}^{*}$ (the Ideal Government's Utility).

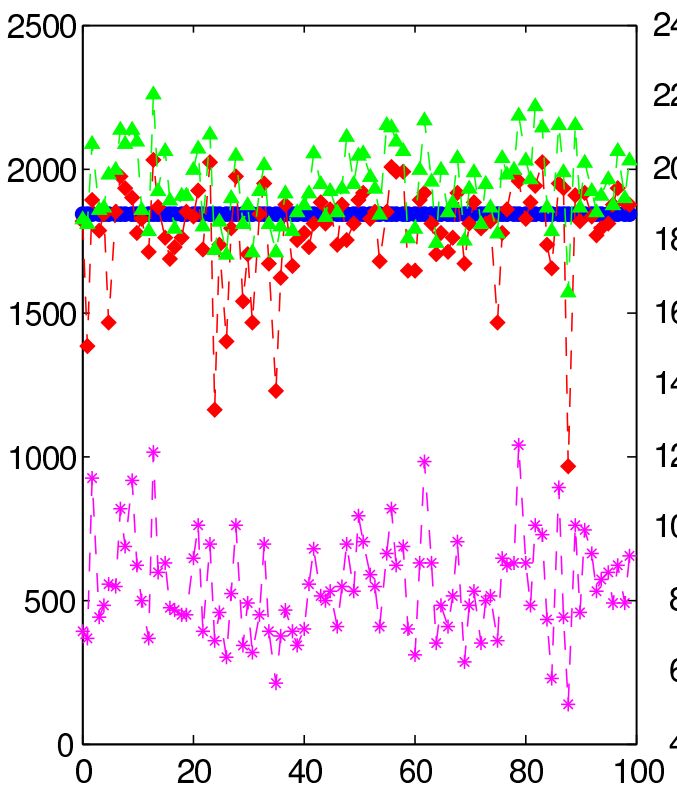

(a) Iteration, with $I=0$

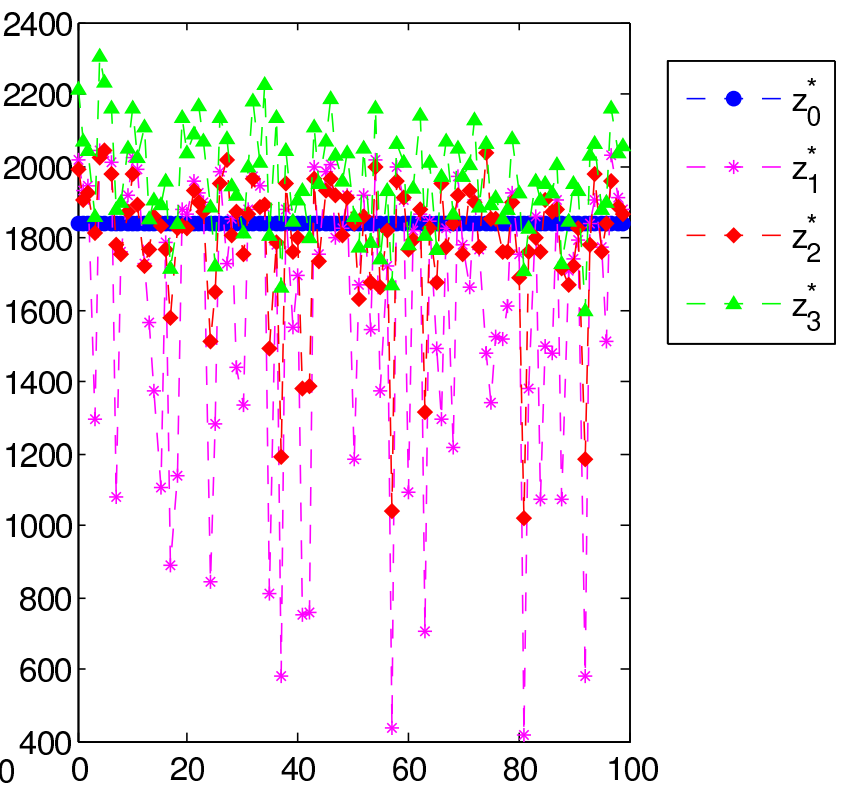

(b) Iteration, with $I=\mu_{\text {outage }} \tilde{r}$

Figure 3: Monte Carlo simulation-based comparison results between the Best Response Policy and two Fixed Inventory Level Policies, and the Best Response Policy, respectively.

Observation 1: On average, using the Best Response Policy against disruptions is much better than using the Fixed Inventory Level Policy.

Table 3 shows that the average percent gains of the government's utility due to using the Best Response Policy, compared with the Fixed Inventory Level Policy, are $260.17 \%$, with the fixed inventory level equal to zero (Policy A) and $21.11 \%$, and with the fixed inventory level equal to 
Table 3: Numerical study of the percent gain of the government's utility using the Best Response Policy compared to the Fixed Inventory Level Policy A and B, and the percent loss of the government's utility using the Best Response Policy compared with the Ideal Government's Utility.

\begin{tabular}{c|c|c}
\hline Difference in Performance & Value for $\lambda=1$ & Value for $\lambda=1.5$ \\
\hline \hline $\bar{\omega}_{A}\left(\omega_{A}=\left(z_{2}^{*}-z_{1}^{A *}\right) /\left|z_{1}^{A *}\right|\right)$ & $286.55 \%$ & $345.58 \%$ \\
$\bar{\omega}_{B}\left(\omega_{B}=\left(z_{2}^{*}-z_{1}^{B *}\right) /\left|z_{1}^{B *}\right|\right)$ & $18.44 \%$ & $23.37 \%$ \\
$\bar{\omega}_{23}\left(\omega_{23}=\left(z_{2}^{*}-z_{3}^{*}\right) /\left|z_{3}^{*}\right|\right)$ & $-9.39 \%$ & $-15.71 \%$ \\
\hline
\end{tabular}

$\mu_{\text {outage }} \cdot \tilde{r}$ (Policy B). Furthermore, assuming $\omega_{i}$ follows a normal distribution with unknown variance, and using the corresponding formula $\left[\bar{\omega}_{i}-\frac{1.645 \cdot s_{\omega_{i}}}{\sqrt{100}}, \bar{\omega}_{i}+\frac{1.645 \cdot s_{\omega_{i}}}{\sqrt{100}}\right], i=A, B$, we calculate the $90 \%$ confidence interval (CI) for the true means of $\omega_{A}$ and $\omega_{B}$ :

- A $90 \%$ CI for the true mean of $\omega_{A}$ is $[241.20 \%, 331.90 \%]$;

- A $90 \%$ CI for the true mean of $\omega_{B}$ is $[13.60 \%, 23.28 \%]$.

The simulation results imply that the government can gain a significant increase in the utility, if the government prepares for supply chain disruptions based on the Best Response Policy presented in this paper, rather than on the Fixed Inventory Level Policy.

Recall that, in order to calculate the inventory protection level $I^{*}$ of the Best Response Policy, we consider a triangle distribution for the length of outage; but under the Fixed Inventory Level Policy with $I=\mu_{\text {outage }} \cdot r$, only the mean outage length is taken into consideration (i.e., less information about the length of the outage is considered). Therefore, it is reasonable and intuitive to observe that the government experiences utility gain by using the Best Response Policy in place of the Fixed Inventory Level Policy.

Note that, if we consider the range length of a random variable $Q(Q \in\{X, L, R\})$ as $\left(Q_{U L}-Q_{L L}\right) \cdot \lambda$, i.e., $Q \in\left[\frac{Q_{L L}+Q_{U L}}{2}-\frac{Q_{U L}-Q_{L L}}{2} \cdot \lambda, \frac{Q_{L L}+Q_{U L}}{2}+\frac{Q_{U L}-Q_{L L}}{2} \cdot \lambda\right]$, we will have the baseline case corresponding to $\lambda=1$. We keep $Q_{M O}$ constant, but increase the value of $\lambda$ from $\lambda=1$ to $\lambda=1.5$; i.e., the ranges of the three randomly generated parameters are enlarged. Based on our simulation results (as shown in Table 3), we have:

- $\bar{\omega}_{A}=345.58 \%$ and a $90 \%$ CI for the true mean of $\omega_{A}$ is $[261.96 \%, 429.19 \%]$;

- $\bar{\omega}_{B}=23.37 \%$ and a $90 \%$ CI for the true mean of $\omega_{B}$ is $[18.58 \%, 28.16 \%]$.

Observation 2: On average, the more uncertainties associated with disruptions (higher $\lambda$ value), the more gain the government experiences by using the Best Response Policy against disruptions, as compared to using the Fixed Inventory Level Policy.

Increasing the range of the outage length affects the inventory protection level $I^{*}$ of the Best Response Policy; however, since we used a symmetric triangle distribution of the outage length (i.e., the mean outage length does not change with the value of $\lambda$ ), the Best Response Policy will perform even better, as compared to the Fixed Inventory Level Policy, when there are more uncertainties associated with disruptions.

Table 3 shows that, compared with the Ideal Government's Utility, the average percent loss due to using the Best Response Policy $\left|\bar{\omega}_{23}\right|$, is $15.71 \%$ (and a $90 \%$ CI for the true mean of $\omega_{23}$ is $[-18.75 \%,-12.67 \%]$ ) when $\lambda=1.5$, and $\left|\bar{\omega}_{23}\right|=9.39 \%$ (and a $90 \%$ CI for the true mean of $\omega_{23}$ is $[-10.71 \%,-8.07 \%])$ when $\lambda=1$.

Observation 3: On average, the government's utility under the Best Response Policy is close to the Ideal Government's Utility.

The above two average errors, $\left(\bar{\omega}_{23}=-9.39 \%\right.$ for the case of $\lambda=1$ and $\bar{\omega}_{23}=-15.71 \%$ for the case of $\lambda=1.5$ ), are almost certainly smaller than the potential estimation errors in the input data, many of which can only be roughly estimated. Our observation suggests that the potential benefits of using a more accurate input data set is positive, but may not be worth the cost. In short, if the government uses our inventory protection model against disruptions, roughly knowing the input data might be adequate. 
Observation 4: On average, the fewer uncertainties associated with disruptions, the less percent loss the government experiences by using the Best Response Policy, as compared with the Ideal Government's Utility.

Intuitively, when there are fewer uncertainties associated with disruptions, the Best Response Policy achieves results closer to the Ideal Preparation Policy.

\subsection{Impact of Capacity Backup Mechanism on Optimal Government's utility}

In this section, we compare the capacity backup protection policy, based on our Capacity Backup model, with the no-capacity-backup-preparation policy that the government may use. In the following, we call such policy No Capacity Backup Policy, and the corresponding numerical model to calculate the government's utility $\left(z_{1}\right)$ is as follows:

$$
\max _{r} z_{1}=\frac{r}{r+R} \cdot v-m \cdot(L \cdot r) .
$$

\subsubsection{Capacity Backup Numerical Model}

Based on the baseline case described in Section 3.1 and Equation (2), we have the following Capacity Backup numerical model to calculate the government's utility $\left(z_{0}\right)$ :

$$
\begin{aligned}
\max _{I, r} z_{0}(r)= & \frac{r}{r+R} \cdot v-\alpha \cdot r \cdot(E[X]+E[L]) \\
& -\beta \cdot r \cdot\left\{\sum_{l=L_{L L}}^{L_{M O}} f_{L}^{(1)}(l) \cdot\left(\sum_{d=D_{L L}}^{D_{M O}}(l-d) \cdot f_{D}^{(1)}(d)+\sum_{d=D_{M O}+1}^{D_{U L}}(l-d) \cdot f_{D}^{(2)}(d)\right)\right. \\
& \left.\quad+\sum_{l=L_{M O}+1}^{L_{U L}} f_{L}^{(2)}(l) \cdot\left(\sum_{d=D_{L L}}^{D_{M O}}(l-d) \cdot f_{D}^{(1)}(d)+\sum_{d=D_{M O}+1}^{D_{U L}}(l-d) \cdot f_{D}^{(2)}(d)\right)\right\} \\
& -m \cdot r \cdot E[D],
\end{aligned}
$$

where $E[Q]=\frac{Q_{L L}+Q_{M O}+Q_{U L}}{3}$ for $Q \in\{X, L, D\}, f_{Q}^{(1)}(q)=\frac{2\left(q-Q_{L L}\right)}{\left(Q_{U L}-Q_{L L}\right)\left(Q_{M O}-Q_{L L}\right)}$ and $f_{Q}^{(2)}(q)=\frac{2\left(Q_{U L}-q\right)}{\left(Q_{U L}-Q_{L L}\right)\left(Q_{U L}-Q_{M O}\right)}$ for $Q \in\{L, D\}$.

The optimal expected government's utility $z_{0}^{*}\left(r^{*}\right)$ is a function of the government's optimal resource $r^{*}$, which is calculated under the baseline case as shown in Table 2. In the following, we refer the policy, making use of both the capacity backup and the government's optimal resource level, as the Best Response Policy.

To examine whether the Best Response Policy performs well under randomly generated scenarios, we introduce $z_{2}^{*}$ as the government's utility, with the Best Response Policy for each randomly generated set of parameters $(X, L, D$, and $R)$ :

$$
z_{2}^{*}=\frac{r^{*}}{r^{*}+R} \cdot v-\alpha \cdot r^{*} \cdot(X+L)-\beta \cdot r^{*} \cdot[L-D]^{+}-m \cdot r^{*} \cdot D .
$$

Under each randomly generated set of parameters $(X, L, D$, and $R)$, given that the government adopts the capacity backup preparation mechanism against disruptions, we calculate the optimal government's resource level in order to maximize the government's utility $\left(z_{3}\right)$ :

$$
\max _{r} z_{3}(r)=\frac{r}{r+R} \cdot v-\alpha \cdot r \cdot(X+L)-\beta \cdot r \cdot[L-D]^{+}-m \cdot r \cdot D .
$$

As indicated in Section 3.2, $z_{3}^{*}$ can be considered the Ideal Government's Utility, while the corresponding optimal policy can be viewed as the Ideal Preparation Policy.

In the following, in order to further investigate the quantitative benefit of using the Best Response Policy, we compare the Best Response Policy with the No Capacity Backup Policy and the Ideal Preparation Policy. 


\subsubsection{Experiment Procedure for Capacity Backup Model}

The experiment procedure is as follows:

Step 1: Use Equation (8) to find the government's optimal resource $r^{*}$ for the baseline case (as shown in Table 2); calculate the government's optimal utility $z_{0}^{*}\left(r^{*}\right) ;$ record $r^{*}$ and $z_{0}^{*}$.

Step 2: $\quad$ Randomly generate 100 sets of the following four parameters: $X, L, D$, and $R$.

Step 3: For each set of generated parameters $(X, L, D$, and $R)$, calculate and record the government's utility $z_{1}^{*} \equiv z_{1, r=\hat{r}}^{*}$ according to Equation (7), where $\hat{r}$ is the optimal solution to Equation (7) obtained by using simulation optimization method as shown below:

- Sub-Step 1: Consider the following possible levels of $r: r^{i}=i, \forall i=0,1, \cdots, 800$.

- Sub-Step 2: For each level of $r, r=r^{i}, i=0,1, \ldots, 800$, use the 100 sets of four randomly generated parameters $(X, L, D$, and $R$ ) and Equation (7) to calculate the corresponding 100 values of $z_{1, r=r} i$; record the average value $\bar{z}_{1, r=r i}$.

- Sub-Step 3: Compare all $\bar{z}_{1, r=r^{i}}, i=0,1, \ldots, 800$, find $\hat{r} \equiv r^{i *}=\arg \max _{r^{i}}\left\{\bar{z}_{1, r=r i}\right\}$, and record $\hat{r}$.

Step 4: For each set of the generated parameters, calculate and record the government's utility $z_{2}^{*} \equiv z_{2, r=r^{*}}^{*}$ according to Equation (9), and the optimal government's utility $z_{3}^{*}$ according to Equation (10).

Step 5: Under each set of generated parameters, calculate and record:

- the value $\omega=\left(z_{2}^{*}-z_{1}^{*}\right) /\left|z_{1}^{*}\right|$, representing the percent increase of utility that the government would experience if she invests in capacity backup preparedness;

- the value $\omega_{23}=\left(z_{2}^{*}-z_{3}^{*}\right) /\left|z_{3}^{*}\right|$, representing the difference between the government's utility using the Best Response Policy and the Ideal Government's Utility.

Step 6: Find the sample means of $\omega$ and $\omega_{23}$, and denote them as $\bar{\omega}$ and $\bar{\omega}_{23}$, respectively.

Step 7: Find the sample standard deviations of $\omega$ and $\omega_{23}$, and denote them by $s_{\omega}$ and $s_{\omega_{23}}$, respectively.

\subsubsection{Simulation Result}

The simulation results of the experiment are shown in Table 4. Figure 4 demonstrates $z_{1}^{*}$ (the government's utility using the No Capacity Backup Policy), $z_{2}^{*}$ (the government's utility using the Best Response Policy), and $z_{3}^{*}$ (the Government's Ideal Utility).

Table 4: Numerical study of the percent gain of the government's utility using the Best Response Policy, compared with the No Capacity Backup Policy and the percent loss compared with the Ideal Government's Utility.

\begin{tabular}{c|c|c}
\hline Difference in Performance & Value for $\lambda=1$ & Value for $\lambda=1.5$ \\
\hline \hline $\bar{\omega}\left(\omega=\left(z_{2}^{*}-z_{1}^{*}\right) /\left|z_{1}^{*}\right|\right)$ & $69.41 \%$ & $109.11 \%$ \\
$\bar{\omega}_{23}\left(\omega_{23}=\left(z_{2}^{*}-z_{3}^{*}\right) /\left|z_{3}^{*}\right|\right)$ & $-1.07 \%$ & $-3.11 \%$ \\
\hline
\end{tabular}

Observation 5: On average, using the Best Response Policy against disruptions demonstrates better results when compared with the No Capacity Backup Policy.

The average percent gain of the government's utility, due to investment in capacity backup preparedness, is $69.41 \%$ (as shown in Table 4 ), while a $90 \%$ CI for the true mean of $\omega$ is $[40.19 \%, 98.62 \%]$, which implies that, if government prepares against supply chain disruptions using the capacity backup model presented in Section 2, the increase in the government's utility is significant.

In the baseline case we use $\lambda=1$ to determine the ranges of four randomly generated parameters. Here, we keep $Q_{M O}(Q \in\{X, L, D, R\})$ constant, but increase the value of $\lambda$ to $\lambda=1.5$; i.e., the ranges of all four randomly generated parameters are enlarged. Based on our simulation results for the case of $\lambda=1.5$, we show that $\bar{\omega}=109.11 \%$ (as shown in Table 4), and that a $90 \%$ CI for the true mean of $\omega$ is $[24.93 \%, 193.30 \%]$. 


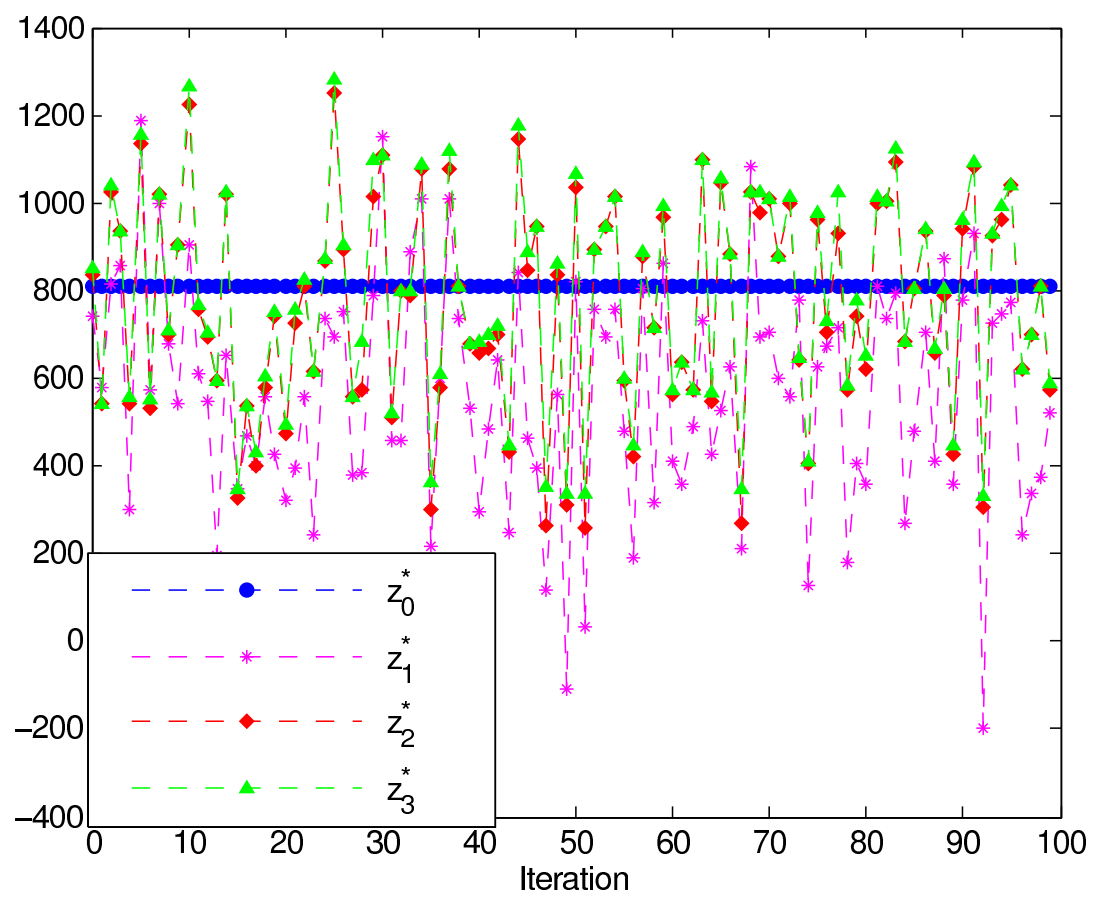

Figure 4: Monte Carlo simulation-based comparison results between the Capacity Backup Policy and No Capacity Backup Policy, and the Best Response Policy, respectively.

Observation 6: On average, the more that uncertainties are associated with disruptions, the more gain the government experiences by using the capacity backup protection mechanism, compared with using no capacity backup.

By comparing $z_{2}^{*}$ with $z_{3}^{*}$, we investigate the difference between using the Best Response Policy and using the Ideal Preparation Policy. Our simulation results shows that:

- when $\lambda=1, \bar{\omega}_{23}=-1.07 \%$ (as shown in Table 4) and a $90 \% \mathrm{CI}$ for the true mean of $\omega_{23}$ is $[-1.33 \%,-0.81 \%]$

- when $\lambda=1.5, \bar{\omega}_{23}=-3.11 \%$ (as shown in Table 4) and a $90 \%$ CI for the true mean of $\omega_{23}$ is $[-3.91 \%,-2.30 \%]$.

Observation 7: On average, the government's utility under the Best Response Policy is very close to the Ideal Government's Utility.

Note that, under both policies, the government uses the capacity backup against disruptions. The only difference is in the government's resource level under each policy, which may have relatively little impact on the government's utility.

\section{CONCLUSION AND FUTURE RESEARCH DIRECTIONS}

In war zones, a government's performance may be dramatically affected by disruptions to military supply chains. Fortunately, the impact of disruptions may be mitigated by pre-disruption preparation mechanisms. In this paper, we have investigated two protection mechanisms (inventory protection mechanism and capacity backup protection mechanism), and their impact on the government's total expected utility in wargames.

Under our inventory protection model, the government's objective is to maximize the total expected utility by balancing the trade-off between the protection cost of inventory preparation and the warzone disadvantage caused by military supply chain disruptions. We conducted a set of Monte Carlo simulation-based experiments to compare the Best Response Policy with the Fixed Inventory Level Policy. We observed that, on average, using the Best Response Policy against disruptions is much 
better than using the Fixed Inventory Level Policy. Furthermore, we found that when there were more uncertainties associated with disruptions, the government would experience greater gains by using the Best Response Policy, as compared with the Fixed Inventory Level Policy. We also compared the Best Response Policy with the Ideal Preparation Policy and found that the government's utility of using the Best Response Policy is close to the utility when using the Ideal Preparation Policy, which implies that knowing only the approximate input data might be adequate, since the potential benefit of using a more accurate input data set is probably not worth the costs.

Under the capacity backup model, our simulation results show that, on average, using the Capacity Backup Policy against disruptions is much better than using the No Capacity Backup Policy; and furthermore, the government's utility of using the Capacity Backup Policy is very close to the Ideal Government's Utility.

Interesting future research directions in this under-studied field of integrating supply chain risk management with wargames include:

- Combining the inventory protection and capacity backup protection to against disruptions;

- Investigating multiperiod inventory protection plan, integrated with a multi-period wargame;

- Extending the single-resource model to multiple-resource one; and

- Extending our single capacity backup model to a more complex one that considers multiple capacity backup providers; furthermore, selecting suitable capacity backup supplier(s) from the candidate pool according to capacity flexibility, capacity cost, and reliability may be considered.

\section{ACKNOWLEDGMENTS}

This research was supported by the United States Department of Homeland Security through the National Center for Risk and Economic Analysis of Terrorism Events (CREATE) under grant number 2007-ST-061-000001. Any opinions, findings, conclusions, or recommendations in this document are those of the authors and do not necessarily reflect views of the United States Department of Homeland Security.

\section{REFERENCES}

Chopra, S., G. Reinhardt, and U. Mohan. 2007. The importance of decoupling recurrent and disruption risks in a supply chain. Naval Research Logistics 54 (5): 544-555.

Chopra, S., and M. Sodhi. 2004. Managing risk to avoid supply-chain breakdown. MIT Sloan management review 46 (1): 53-61.

Christopher, M., and H. Lee. 2004. Mitigating supply chain risk through improved confidence. International Journal of Physical Distribution and Logistics Management 34 (5): 388-396.

Christopher, M., and H. Peck. 2004. Building the resilient supply chain. International Journal of Logistics Management 15 (2): 1-13.

Encyclopedia of the Nations 2008. Hondras economy. Available at http: / / www . nationsencyclopedia.com/Americas/Honduras-ECONOMY . html. Accessed on April 12, 2010.

Hopp, J. W., M. Liu, and Z. Liu. 2010. Protecting supply chain networks against catastrophic failures. Working paper, Department of Operations and Information Management, Wisconsin School of Business, University of Wisconsin-Madison.

Juttner, U., H. Peck, and M. Christopher. 2003. Supply chain risk management: Outlining an agenda for future research. International Journal of Logistics: Research and Applications 6 (4): 197-210.

Sheffi, Y. 2002. Supply chain management under the threat of international terrorism. International Journal of Logistics Management 12 (1).

Sheffi, Y., J. Rice, J. J. Fleck, and F. Caniato. 2003. Supply chain response to global terrorism: A situation scan. Proceedings of EUROMA-POMS Join International Conference. Available at http: //web.mit.edu/scresponse/. Accessed on April 12, 2010.

UK Agricultural Biodiversity Coalition 2004. Indian ocean tsunamis devastate fisherfolk. Available at http: //www. ukabc.org/tsunamis.htm. Accessed on April 12, 2010.

Zhuang, J., and V. M. Bier. 2007. Balancing terrorism and natural disasters- Defensive strategy with endogenous attack effort. Operations Research 55 (5): 976-991. 


\section{AUTHOR BIOGRAPHIES}

SHILAN JIN is a Ph.D student in Industrial and Systems Engineering at the University at Buffalo. Her research interests include game theory, supply chain risk management, and health care.

ZIGENG LIU is an Assistant Professor of Operations and Information Management at the Wisconsin School of Business. She obtained her Ph.D. in summer 2009 from Northwestern University. Her primary area of research is supply chain risk management. Research papers are under revision for publication in top journals such as Management Science, and a book chapter on "Supply Chain Network Design to Mitigate Effects of Disruption" is in progress.

JUN ZHUANG is an Assistant Professor of Industrial and Systems Engineering at the University at Buffalo, The State University of New York. He has been a faculty member at SUNY Buffalo since he obtained his Ph.D. in summer 2008 from University of Wisconsin-Madison. Dr. Zhuang's long term research goal is to integrate operations research and game theory to better prepare for, mitigate, and manage both natural and man-made hazards. Other areas of interest include health care, transportation, logistics and supply chain management, and sustainability. 